\title{
BWI EM FUNÇÃO DA MALHA DE TESTE
}

\author{
T. F. M. B. DUQUE ${ }^{1}$, C. L. SCHNEIDER ${ }^{1}$, D. B. MAZZINGHY ${ }^{2}$ e V. K. ALVES $^{3}$ \\ ${ }^{1}$ Centro de Tecnologia Mineral - CETEM \\ ${ }^{2}$ Anglo American \\ ${ }^{3}$ VALE S.A.
}

tduque@cetem.gov.br; cschneid@cetem.gov.br; douglas.mazzinghy@angloamerican.com; vladmir.alves@vale.com

Artigo submetido em novembro/2013 e aceito em janeiro/2014

DOI: http://dx.doi.org/10.15628/holos.2014.1842

\section{RESUMO}

O Índice de Trabalho de Bond para moagem em moinhos de bolas $(B W I)$ é medido de acordo com uma metodologia padrão, utilizando equipamentos igualmente padronizados. O ensaio é um ensaio cíclico fechado, e resulta em um único número, o $B W I$, característico do minério, e que é amplamente utilizado para o escalonamento de moinhos de bolas industriais. A medição do $B W I$ geralmente é feita por profissionais treinados, com a ajuda de uma planilha eletrônica tipo Excel para os cálculos necessários antes, durante e após a conclusão do ensaio. A norma para determinação do $B W I$ registrada no INMETRO é a NBR 11376. Embora completamente padronizada, a metodologia para medição do $B W I$ pode ser melhorada, otimizada, e escrutinada. A demanda por medições do BWI tem aumentado significativamente pelo simples fato de um único número conter todas as informações necessárias para o escalonamento de moinhos de bolas, que é a etapa mais energeticamente intensiva em uma planta de processamento de minérios. Esta característica faz do $B W I$ um parâmetro central em campanhas de variabilidade e geometalurgia. Neste trabalho são apresentadas duas importantes propriedades do BWI, sua dependência com a malha de teste e sua relação com a fração inicial passante na amostra do ensaio.

PALAVRAS-CHAVE: Cominuição, Bond, BWI, Caracterização.

\section{BWI AS A FUNCTION OF TEST SIEVE}

\begin{abstract}
The Bond Ball Work Index (BWI) is measured according to a standard test using standardized equipment. The test is a closed-cycle test, and results in a single number, the BWI, characteristic of the ore, and which is used extensively for scaling up industrial mills. The measurement of the BWI is usually done by trained personnel with the help of an Excel spreadsheet for the required calculations that take place before, during and after completing the test. The Brazilian standard for determining the BWI is registered in INMETRO as norm NBR 11376. Although completely standardized, the
\end{abstract}

methodology for measuring BWI can be improved, optimized, and scrutinized. The demand for BWI measurements has increased significantly because a single number contains all information necessary for the scaling up of ball mills, which is the most energy-intensive step in an ore processing plant. This property makes the BWI a preferred parameter in variability campaigns and geometallurgy. This work presents two important properties of the BWI, its dependence on the test sieve opening and its relationship with the initial fraction of passing material in the test sample.

KEYWORDS: Comminution, Bond, BWI, Characterization. 


\section{INTRODUÇÃO}

Índices de trabalho de moagem se referem a parâmetros derivados de ensaios de similaridade realizados com moinhos padrão, nominalmente o índice de trabalho de moagem de bolas e o índice referente à moagem com barras. Um terceiro índice existe, nominalmente índice de trabalho de britagem, que por sua vez não é baseado em um ensaio de similaridade e sim em um ensaio mais fundamental, de resistência à fratura por impacto. Todos estes índices são bem conhecidos e utilizados na prática básica de engenharia, e são basicamente parâmetros de escalonamento de moinhos e britadores, embora muitos outros usos tenham sido praticados corriqueiramente em cálculos metalúrgicos (operação de plantas de processamento e geometalurgia em geral).

Os índices e os sistemas de escalonamento baseados nestes índices foram desenvolvidos no início do século XX por Fred Bond, um brilhante engenheiro americano que na época trabalhava para a empresa Allis-Chalmers, fabricante de moinhos e britadores entre outros equipamentos. A história é bem conhecida e os diversos índices são conhecidos há décadas como índices de Bond. O termo índice de trabalho advém de uma tradução literal do Work Index de Bond e, no Brasil, existe inclusive uma norma ABNT para regulamentar a medição do índice de trabalho em moinhos de bolas, registrada no INMETRO como NBR 11376. Os motivos da padronização deste ensaio, especificamente, não são claros, já que não há comercialização de produtos com índice de trabalho de moagem de bolas conhecido. A ideia, por outro lado, é atraente, e seria interessante se houvesse no mercado amostras de referência certificadas de índice de trabalho de moagem de bolas, para calibração de laboratórios em empresas de mineração de prestação de serviços. A utilização do índice de trabalho de Bond de bolas (BWI, Ball Work Index) em campanhas de geometalurgia tem aumentado consideravelmente, e este fato é percebido pela oferta de determinações expeditas por empresas tradicionais de prestação de serviços metalúrgicos como, por exemplo, a SGS que oferece o índice de trabalho comparativo, baseado em um ensaio simplificado com uma amostra de BWI conhecido, o JKTech, que oferece um $B W I$ previsto baseado em um número de ciclos menor (três ciclos em geral). No Brasil, a Vale tem utilizado o método referido como Anaconda proposto por Yap, Sepulveda e Juaregui (1982) ou, alternativamente, a previsão do $B W I$ a partir de um ensaio simplificado para determinação de parâmetros e da simulação do ensaio completo com estes parâmetros (Alves e Schneider, 2010) com um software desenvolvido para este fim (BondAccel).

O método Anaconda pode (e possivelmente deve) ser utilizado como um método comparativo. Assim, em uma campanha com 150 determinações, que são comuns em projetos com geometalurgia (Schneider et al., 2012), o BWI é determinado para um lote menor de amostras (em geral vinte ou trinta amostras) utilizando-se o procedimento padrão, e o procedimento simplificado (Anaconda). Um parâmetro de calibração é determinado para esta sub-amostra e subsequentemente o $B W I$ é determinado para as amostras restantes somente pelo método simplificado. Como em campanhas de geometalurgia várias amostras (dezenas) requerem a determinação do $B W I$ pelo método padrão, é interessante otimizar o procedimento para determinação do BWI. Surgem com isso questões sobre a otimização do procedimento de ensaio, como por exemplo, qual é o número de rotações do primeiro ciclo? Isso faz diferença? Qual a malha 
de teste mais apropriada? Esta questão também aparece em estudos sobre otimização de plantas de moagem.

Recentemente, no CETEM, estão surgindo aplicações para técnicas de rochagem na agricultura e para processamento de passivos ambientais, como o passivo da região de Criciúma, SC. Nestes dois casos, o custo da moagem é um fator crítico, podendo facilmente inviabilizar projetos sensíveis aos custos de produção devido ao baixo valor de mercado dos produtos. 0 sistema de escalonamento de moinhos de bolas de Bond foi ajustado e corrigido diversas vezes desde 1941 (ano da sua publicação), especialmente pela introdução de fatores de correção. Muito deste trabalho foi desenvolvido por Chester Rowland, outro engenheiro da Allis-Chalmers, pupilo de Fred Bond no projeto de plantas de britagem e moagem. Rowland por sua vez, parece ter sido muito mais fluente e produziu diversas publicações técnicas, sendo que na sua última versão do método de escalonamento de Bond Rowland (2006) foi categórico em afirmar que o BWI deve ser medido na malha de teste que reflete a granulometria desejada no produto do moinho que está sendo escalonado, colocando um fim na existência de um $B W I$ medido em uma malha de teste padrão, até então $150 \mu \mathrm{m}$. Os objetivos deste trabalho são determinar o BWI em diversas malhas de teste, revelando o comportamento deste importante parâmetro em função da aplicação a ser implementada, e demonstrar como o número inicial de rotações do ensaio pode ser estimado a partir de um WI presumido.

\section{METODOLOGIA}

\subsection{Previsão do $B W I$ de Bond para qualquer malha de teste}

Uma questão recorrente em projetos de cominuição diz respeito à dependência do $B W I$ na malha de teste. Em princípio, o BWI é um parâmetro característico do material, porém, fosse assim, - $B W I$ seria constante para todas as malhas de teste se os minérios fossem homogêneos com respeito ao tamanho de partícula. Porém, os minérios em geral não são homogêneos, nem o $B W I$ é independente da malha de teste. Recentemente uma empresa de mineração requisitou a medição do $B W I$ de uma amostra de minério de titânio na malha de 850 micrômetros, que é uma abertura um tanto fora do padrão. Valores de BWI medidos utilizando o procedimento padrão (ensaio padrão de Bond) foram medidos em duplicata. Como a malha de teste requerida é relativamente alta, foi feito um estudo adicional para testar uma metodologia de previsão do BWI a partir de ensaios em malhas reconhecidamente padrão, ou em torno da malha padrão, que é de 150 micrômetros. Assim, foram realizados ensaios adicionais nas malhas 212, 150 e 106 micrômetros. Os resultados de todos os ensaios são apresentados de forma sumária na Tabela 1.

Tabela 1 - Resultado dos ensaios de BWI de Bond, metodologia padrão, nas diversas malhas de teste estudadas, com a duplicata na malha de 850 micrômetros.

\begin{tabular}{ccccc}
\hline Malha de teste $(\boldsymbol{\mu m})$ & $\mathbf{A 8 0}(\boldsymbol{\mu m})$ & $\mathbf{P 8 0}(\boldsymbol{\mu m})$ & Mob $(\mathrm{g} / \mathbf{r o t})$ & $\boldsymbol{B W I}(\mathbf{k W h} / \mathbf{t})$ \\
\hline 850 & 2302.3 & 729.8 & 5.42 & 16.05 \\
850 & 2297.1 & 721.3 & 5.39 & 15.93 \\
212 & 2347.7 & 179.9 & 2.22 & 13.75 \\
150 & 2358.0 & 118.5 & 1.68 & 14.16 \\
106 & 2350.1 & 86.3 & 1.39 & 14.69 \\
\hline
\end{tabular}


Qualquer valor de $B W I$ é uma função da malha de teste $M T, P 80$ e $A 80$, em micrômetros, e do valor de GPR que é o peso, em gramas, de material produzido por revolução do moinho com tamanhos abaixo da malha de teste a partir do material que se encontrava acima da malha de teste na alimentação do moinho. Deve-se notar que o ensaio padrão de Bond nada mais é do que a determinação do número de rotações necessárias para que a carga circulante (oversize da peneira de teste) iguale $250 \%$ da alimentação fresca. A relação entre as diversas variáveis do ensaio e o $B W l$ é mostrada na eq. (1) abaixo:

$B W I=\frac{1.1 \times 44.5}{M T^{0.23} \times G P R^{0.82} \times 10 \times\left(\frac{1}{\sqrt{P 80}}-\frac{1}{\sqrt{A 80}}\right)}$

A eq. (1) é a fórmula do $B W I$ para a condição de convergência do ensaio padrão de Bond. Para a previsão do $B W I$ em qualquer malha de teste, postula-se que tanto os valores de GPR (Gramas Por Rotação) e de $P 80$ possam ser estimados acuradamente a partir do valor da malha de teste $M T$. Para tanto, uma correlação entre estes valores deve ser estabelecida. Sabe-se que o valor de $P 80$ é, em geral, correlacionado à malha de teste $M T$ da seguinte forma:

$P 80=\frac{M T}{\sqrt{2}}=0.707 \times M T$

Um gráfico mostrando a correlação obtida nos ensaios com a amostra de minério de titânio é mostrado na Figura 1.

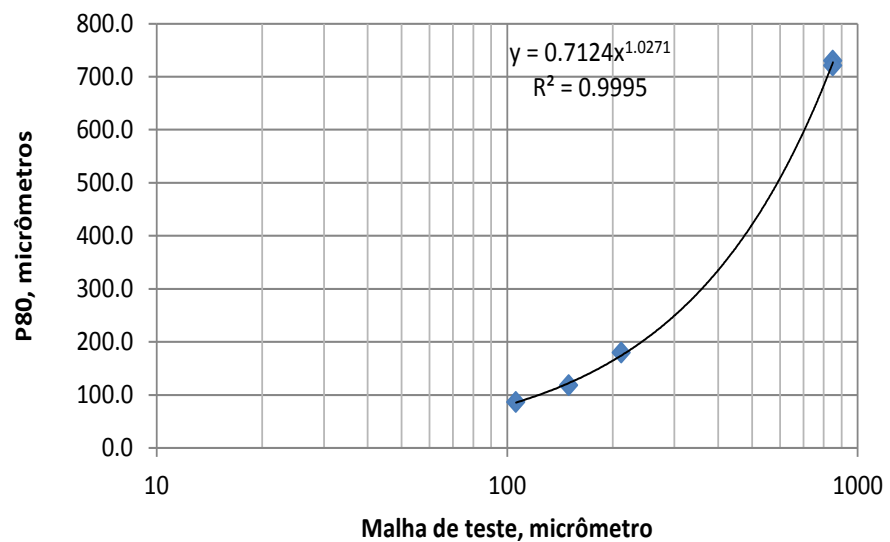

Figura 1 - Correlação entre a malha de teste e o valor de $P 80$ para a amostra de minério de titânio.

Pode-se ver na Figura 1 que a correlação obtida é muito próxima da correlação de praxe da eq. (2). O coeficiente de correlação é altíssimo (0.9995), indicando que o $P 80$ pode ser estimado a partir do valor da malha de teste com bastante acurácia. É de se esperar que exista, sempre, uma boa correlação entre a abertura da malha de teste e o valor do $P 80$, embora a inclinação da distribuição granulométrica de cada produto não seja necessariamente a mesma para todos os materiais, e isso se deve ao fato de o valor de $P 80$ estar sempre nas imediações da abertura da malha de teste, por definição. Da mesma forma, uma correlação entre o valor de GPR e a malha de teste pode ser estabelecida, como mostrado na Figura 2. 


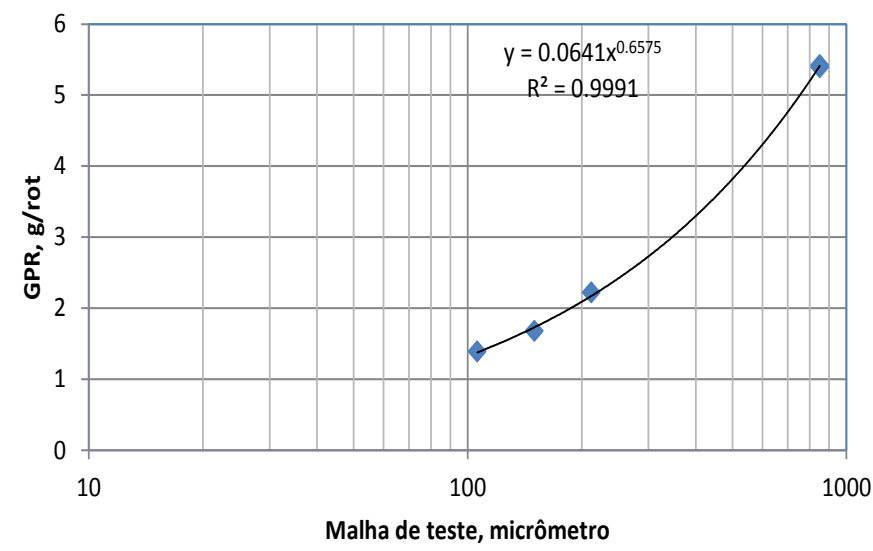

Figura 2 - Correlação entre a malha de teste e o valor de GPR para a amostra de minério de titânio.

Novamente a correlação é altamente satisfatória. Este resultado não é surpreendente. Espera-se que quanto maior a malha de teste, mais grosseira deve ser a alimentação do moinho de teste na condição de convergência $(C C=250 \%)$. É bem sabido que a taxa de moagem aumenta com o tamanho de partícula em moinhos de bolas, contanto que as bolas sejam suficientemente grandes para a moagem. No ensaio de Bond, esta condição é praticamente garantida já que o top size da alimentação é de 3,36 mm e o diâmetro da bola maior da carga de teste é de $36,5 \mathrm{~mm}$. No entanto, deve-se ter em mente que para minérios extremamente tenazes, há a possibilidade de a moagem do ensaio de Bond entrar na região de quebra anormal, e nesta situação a correlação da Figura 2 não é válida. Com estas correlações estabelecidas, o BWI da amostra de minério de titânio pode ser estimado para qualquer malha de teste, usando:

$$
B W I=\frac{1.1 \times 44.5}{M T^{0.23} \times\left(0.0641 \times M T^{0.0641}\right)^{0.82} \times 10 \times\left(\frac{1}{\sqrt{0.7124 \times M T^{1.0271}}}-\frac{1}{\sqrt{A 80}}\right)}
$$

Dado que o valor de $A 80$ é constante e não varia com a malha de teste. Utilizando-se estas correlações, e um $A 80$ médio de $2331 \mu \mathrm{m}$ para esta amostra de minério de titânio (notar que a eq. (3) só é válida para esta amostra de minério de titânio), os resultados de BWI estimados desta forma são mostrados na Tabela 2.

Tabela 2 - Valores de $B W I$ estimados da amostra de minério de titânio em diversas malhas de teste.

\begin{tabular}{ccccc}
\hline MT $(\mu \mathrm{m})$ & $\boldsymbol{A} 80(\mu \mathrm{m})$ & $\boldsymbol{P 8 0 , \mu \mathrm { m }}$ & Mob, g/rot & WI, kWh/t \\
\hline 2380 & 2331 & 2093.2 & 10.6404 & 102.84 \\
2000 & 2331 & 1750.7 & 9.49042 & 42.235 \\
1680 & 2331 & 1463.7 & 8.4625 & 28.3711 \\
1410 & 2331 & 1222.6 & 7.54171 & 22.3348 \\
1190 & 2331 & 1027.1 & 6.74575 & 19.1328 \\
1000 & 2331 & 859.1 & 6.0167 & 17.1149 \\
850 & 2331 & 727.0 & 5.40693 & 15.8767 \\
710 & 2331 & 604.3 & 4.80353 & 14.9543 \\
595 & 2331 & 504.0 & 4.27664 & 14.3536 \\
500 & 2331 & 421.5 & 3.81444 & 13.9687 \\
420 & 2331 & 352.4 & 3.4013 & 13.7349
\end{tabular}




\begin{tabular}{lllll}
350 & 2331 & 292.2 & 3.01705 & 13.6158 \\
297 & 2331 & 246.9 & 2.70829 & 13.5967 \\
250 & 2331 & 206.8 & 2.41826 & 13.6512 \\
210 & 2331 & 172.9 & 2.15634 & 13.7728 \\
180 & 2331 & 147.6 & 1.9485 & 13.9294 \\
150 & 2331 & 122.4 & 1.72838 & 14.1681 \\
125 & 2331 & 101.5 & 1.53312 & 14.4598 \\
106 & 2331 & 85.7 & 1.37561 & 14.7661 \\
90 & 2331 & 72.4 & 1.2353 & 15.1079 \\
75 & 2331 & 60.1 & 1.09575 & 15.5315 \\
63 & 2331 & 50.2 & 0.97707 & 15.9777 \\
53 & 2331 & 42.0 & 0.87211 & 16.4589 \\
44 & 2331 & 34.7 & 0.77166 & 17.0201 \\
38 & 2331 & 29.9 & 0.70075 & 17.4935 \\
\hline
\end{tabular}

Estes resultados são mostrados de forma resumida na Figura 3, abaixo. Como uma segunda alternativa para estudos futuros, correlações foram determinadas somente a partir dos ensaios de $B W I$ nas malhas em torno da malha padrão, ou seja, 106, 150 e 212 micrômetros. Os resultados obtidos a partir desta correlação também são mostrados na Figura 3.

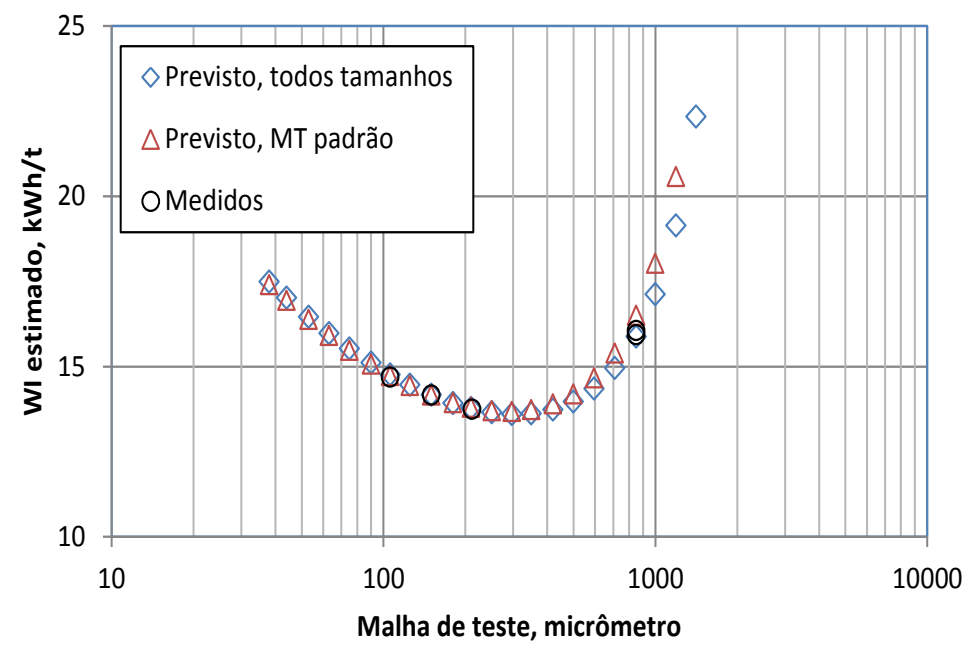

Figura 3 - Valores estimados de BWI (losangos) a partir das correlações obtidas, valores estimados (triângulos) somente a partir das três malhas padrão e valores medidos (círculos).

Pode-se concluir que estimativas de $B W I$ em qualquer malha de teste podem ser feitas com bastante acuracidade a partir de medidas em algumas malhas de teste distintas, em torno da malha de teste padrão, visto que as correlações das Figuras 1 e 2 necessariamente se estendem para tamanhos maiores. A forma da curva na Figura 3 é, de certa forma, surpreendente. Sabe-se que o valor do $B W I$ tende ao infinito à medida que os valores de $A 80$ e $P 80$ se aproximam, por força da forma da eq. (2). Para malhas de teste menores, o BWI decresce monotonicamente. Porém, um mínimo claro ocorre, neste caso, em torno de 300 micrômetros e a partir deste mínimo os valores de BWI aumentam monotonicamente com a redução da malha de teste. Postula-se que este comportamento ocorre para todos os minérios. Vale lembrar que o software BondAccel ${ }^{\mathrm{TM}}$ já previa 
este tipo de comportamento do BWI em função da malha de teste. Substituindo as eqs. (1) e (3) na eq. (2) o $B W I$ para a amostra de $R O M$ de minério de titânio deste projeto fica:

$W I=\frac{1.1 \times 44.5}{1.051 \times M T^{0.76915} \times\left(\frac{\sqrt[4]{2}}{\sqrt{M T}}-\frac{1}{\sqrt{A 80}}\right)}$

Nesta forma fica claro que o termo $\left(\frac{\sqrt[4]{2}}{\sqrt{M T}}-\frac{1}{\sqrt{A 80}}\right)$ tende a zero para valores de $M T$ similares ao $A 80$ da amostra, e, portanto, o BWI tende a infinito à medida que a malha de teste se aproxima do top size da amostra. Ou seja, o termo $\left(\frac{\sqrt[4]{2}}{\sqrt{M T}}-\frac{1}{\sqrt{A 80}}\right)$ é igual a zero quando $M T=\sqrt{2} \times A 80$, que é $3.36 \mathrm{~mm}$ para minérios que seguem a correlação da Figura 1. De qualquer forma, e surpreendentemente, é possível medir o $B W I$ para malhas de teste maiores que o $A 80$ da amostra desde que o $P 80$ resultante seja menor do que $A 80$. Advertência: a previsão do $B W I$ utilizando-se estas correlações não inclui descontinuidades nas características de quebra dos materiais, tais como uma mudança de fratura inter-granular para intra-granular em um tamanho específico. Os valores de $B W I$ previstos assumem que o padrão de fratura é homogêneo no domínio das malhas de teste consideradas. Como o BWI tende ao infinito à medida que a malha de teste se aproxima do top size $(3.36 \mathrm{~mm})$, este resultado pode passar a impressão de que mais energia é requerida para moer menos. No entanto, vale lembrar que a potência requerida é dada pela equação da terceira lei da energia consumida na moagem, de Bond, ou seja:

$P=Q \times W I \times 10 \times\left(\frac{1}{\sqrt{P 80}}-\frac{1}{\sqrt{A 80}}\right)$

De fato, o termo $\left(\frac{1}{\sqrt{P 80}}-\frac{1}{\sqrt{A 80}}\right)$ tende a zero mais rápido que termo $1 /\left(\frac{\sqrt[4]{2}}{\sqrt{M T}}-\frac{1}{\sqrt{A 80}}\right)$ tende ao infinito, e, portanto, quando a malha de teste tende ao top size da amostra, a potência requerida é zero. O gráfico da Figura 4 mostra a evolução da potência requerida, eq. (5), sem fatores de correção aplicados, para moer $Q=1 \mathrm{t} / \mathrm{h}$ da amostra de minério de titânio. Embora o gráfico do $B W I$ nas diversas malhas de teste, Figura 3 , seja em forma de $U$, com um mínimo bem definido, a potência calculada segue a lógica de quanto mais fino o produto, mais potência é necessária parta realizar a moagem.

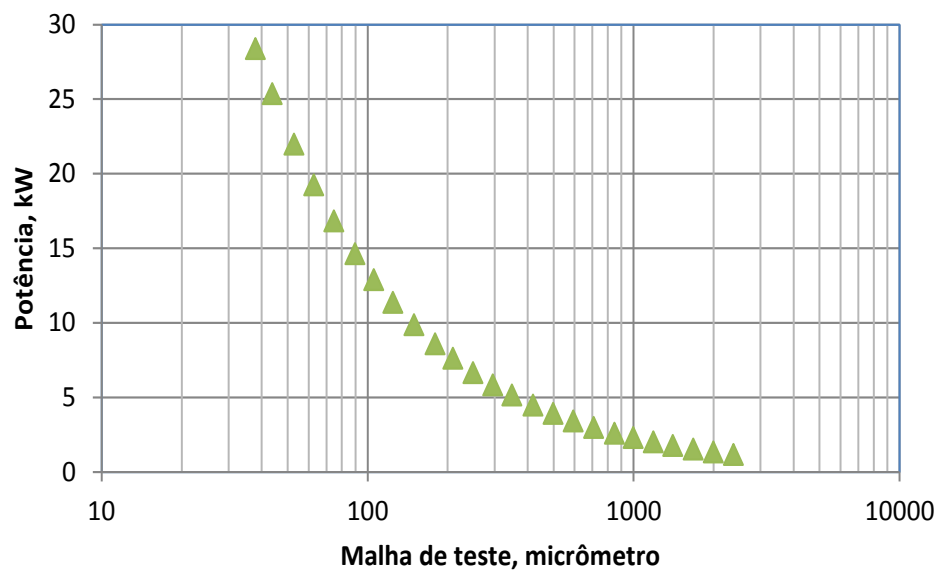

Figura 4 - Potência requerida para moer 1 t/h de amostra de minério de titânio no $P 80$ equivalente à malha de teste. Nenhum fator de eficiência foi aplicado neste cálculo. 
No gráfico da Figura 4 é interessante destacar que, para um moinho de bolas um limite viável para moagem industrial se encontra em torno de $P 80=30$ micrômetros (pelo menos no caso do minério de titânio) e, abaixo desse valor limítrofe, a potência requerida tende rapidamente ao infinito, sempre lembrando que este limite existe de acordo com o escalonamento de Bond, e não se trata de um limite real. A inclinação da curva muda ao longo do eixo da malha de teste, sendo que a taxa de redução de potência passa a ser menos acentuada a partir dos 300 micrômetros, indicando que o $B W I$ mínimo pode ser um $B W I$ ótimo do ponto de vista do melhor custo/benefício de produto de moagem em moinho de bolas.

\subsection{Número de rotações do primeiro ciclo}

A norma indica que o operador deve escolher entre 100 e 200 rotações como o número inicial para o ensaio, sendo que 100 rotações seriam apropriadas para minérios mais friáveis e 200 rotações para minérios mais tenazes. Trata-se na verdade de uma sugestão e o número inicial de rotações é escolhido pelo operador com base em experiências anteriores. Tecnicamente, o ensaio pode ser iniciado com qualquer valor maior do que uma rotação.

Em campanhas de variabilidade, o mesmo tipo de minério é testado centenas de vezes, e nestes casos, o resultado do ensaio pode ser estimado de forma grosseira. Por exemplo, depois de testar vinte amostras, o operador sabe que o resultado do ensaio tem grande probabilidade de estar em torno da média dos ensaios anteriores. Este valor pode ser utilizado para estimar o número de rotações ao final do ensaio, e, pela ausência de estimativa melhor, pode também ser utilizado como o número inicial de rotações do ensaio. Vamos supor que o $B W I$ estimado para o tipo de minério que está sendo testado seja $E B W I$, um valor conhecido. Outras variáveis conhecidas no início do ensaio são o $A 80$, a massa de passante inicial, a massa de alimentação do moinho e a malha de teste $M T$. Com o valor de $M T$ podemos estimar também o valor de $P 80$, usando a eq. (2). Se chamarmos o $P 80$ estimado de EP80, a eq. (1) pode ser escrita como:

$E G P R^{0.82}=\frac{1.1 \times 44.5}{M T^{0.23} \times E B W I \times 10 \times\left(\frac{1}{\sqrt{E P 80}}-\frac{1}{\sqrt{A 80}}\right)}$

Onde EGPR é o valor estimado de GPR ao final do ensaio. O valor de GPR ao final do ensaio é calculado com base em uma carga circulante igual a $250 \%$, na massa de alimentação do moinho $M A$ (determinada no ensaio de densidade aparente) e na fração passante inicial $F P I$ na malha de teste, que é determinada pela distribuição granulométrica da alimentação. Todos os valores são conhecidos no início do ensaio, e:

$$
N I R=\frac{\frac{M A}{3.5} \times(1-F P I)}{E G P R}
$$

Pode ser utilizado como uma estimativa do número inicial de rotações. O uso da eq. (7) praticamente garante que o ensaio convirja com até sete ciclos, dado que a estimativa $E B W I$ esteja próxima do $B W I$ medido ao final do ensaio. Por exemplo, para o minério de titânio deste trabalho, o procedimento inicial do ensaio com a amostra britada com $100 \%$ passante em $3,36 \mathrm{~mm}$, produziu os seguintes dados: densidade aparente $=2,190 \mathrm{~g} / \mathrm{cc}$, massa de alimentação da moagem $M A=$ $1533 \mathrm{~g}$ (calculada com base na densidade aparente), $A 80=2358$ micrômetros. A fração passante inicial em cada malha de teste, $F P I$, é dada pela análise granulométrica completa da alimentação. A estimativa inicial do $B W I$ foi escolhida com base em resultados de medições anteriores, $E B W I=$ $16 \mathrm{kWh} / \mathrm{t}$. A estimativa do $P 80$ ao final do ensaio pode ser feita com base na eq. (2) e no valor da 
malha de teste. O gráfico da Figura 5 mostra o número inicial de rotações para esta amostra de minério de titânio em função da malha de teste. Pode-se ver que, dadas às condições iniciais, quanto menor a malha de teste, maior o número de rotações, e para tamanhos típicos da malha de teste, em torno de 150 micrômetros, o número inicial de rotações é sempre significativamente maior que 200. Ensaios em malhas teste com aberturas grandes podem ser iniciados com menos do que 100 rotações.

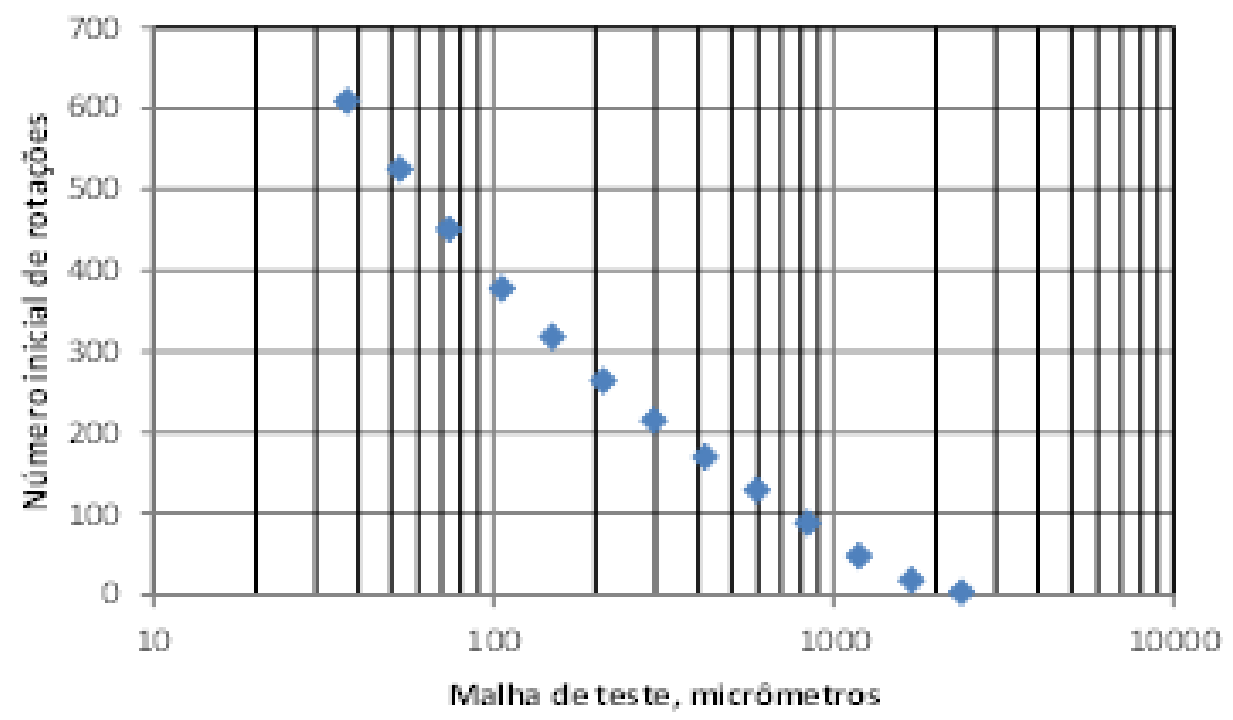

Figura 5 - Número inicial de rotações para esta amostra de minério de titânio em função da malha de teste.

\section{CONCLUSÕES}

O ensaio do $B W I$ pode ser otimizado e para isto basta entender o processo de medição em detalhe. Outra conclusão derivada desta análise é que o $B W I$ pode ser medido para qualquer fração inicial passante na malha de teste menor que $100 \%$, conclusão esta baseda na eq. (7), e no entendimento da natureza do ensaio. Mesmo que a alimentação fresca contenha um micrograma de material retido na malha de teste, existe uma condição na qual a carga circulante de $250 \%$ pode ser atingida, se permitirmos que o número de rotações seja fracional, ou seja, moermos muito pouco material em cada ciclo. O BWI varia com a malha de teste, e apresenta uma curva em forma de $U$, com um mínimo bem definido. Isso não implica que produtos mais grosseiros necessitem mais energia se estivermos do lado direito do mínimo. Trata-se de uma propriedade inerente do índice, e o escalonamento de Bond necessariamente leva em consideração estes detalhes, embora o banco de dados da Allis-Chalmers, à época, provavelmente não devia conter muitas operações com BWls medidos em tamanhos mais grosseiros. Fica a dúvida se o BWI é aplicável para malhas de teste muito acima da malha padrão, ou muito abaixo. Sempre é bom lembrar que o método de escalonamento de Bond é baseado em um ensaio de similaridade. Com isso, o escalonamento é para alimentações finas ( $100 \%$ menor que $3,36 \mathrm{~mm}$ ) e carga circulante de $250 \%$. Claro que existe uma certa flexibilidade com relação ao tamanho da alimentação, já que o $A 80$ é um parâmetro do escalonamento e as bolas utilizadas no ensaio de Bond são grandes o suficiente. Porém, é necessário precauções quanto à zona anormal de quebra quando as partículas ficam muito grandes para serem quebradas pelas bolas da moagem industrial. Este efeito nunca é medido no ensaio de Bond porque o tamanho da alimentação e a carga de bolas são padronizados. 
Hoje em dia existe uma tendência para alimentações mais grosseiras em moinhos de bolas que seriam considerados gigantes na época de Bond e da Allis-Chalmers. Por outro lado, o parâmetro $B W I$ continua sendo um bom estimador de moabilidade e pode ser utilizado em projetos de escalonamento com variabilidade e em aplicações geometalúrgicas, com vantagem, por se tratar de um único número contendo todas as informações que seriam necessárias para caracterizar a moabilidade de uma dada amostra. Porém, sabemos que a moabilidade de qualquer minério necessita de pelo menos dois parâmetros, um para descrever a tenacidade e outro para descrever a produção de finos.

\section{REFERÊNCIAS BIBLIOGRÁFICAS}

1. ALVES, V.K., SCHNEIDER, C.L. Prediction of the Bond Work Index for Variability Analysis, Comminution'10, Cape Town, April 2010.

2. ROWLAND, C.A. Bond's method for selection of ball mills. In: Advances in Comminution. KAWATRA, S.K. (editor), SME, Littleton, New York, p. 385-397, 2006.

3. SCHNEIDER, C.L., MAZZINGHY, D., ALVES, V.K., Bond WI as a parameter for the evaluation of ore variability in current grinding plant design practice. In: Proceedings of the $9^{\text {th }}$ International Conference of Mineral Processing. DOLL, A., KRACHT, W., KURYVENHOVEN, R. (editors). Santiago, 2012, p. 74.

4. YAP, R.F., SEPULVEDA, J.W., JUAREGUI, R. Determination of the Bond Work Index Using an Ordinary Laboratory Batch Mill. In: Design and Installation of Comminution Circuits. MULAR, A.L., JERGENSEN, G.V. (editors). SME, New York, 1982, p. 176. 